\title{
Two-Phase Fluid Flow Experiments Monitored by NMR
}

\author{
Thomas Hiller ${ }^{1}$, Gabriel Hoder ${ }^{2}$, Alexandra Amann-Hildenbrand ${ }^{3}$, Norbert Klitzsch $^{3 *}$, and Norbert Schleifer $^{4}$ \\ ${ }^{1}$ Leibniz Institute for Applied Geophysics, Hannover, Germany \\ ${ }^{2}$ SCK.CEN, Mol, Belgium \\ ${ }^{3}$ RWTH Aachen University, Aachen, Germany \\ ${ }^{4}$ Wintershall Holding GmbH, Barnstorf, Germany
}

\begin{abstract}
We present a newly developed high-pressure nuclear magnetic resonance (NMR) flow cell, which allows for the simultaneous determination of water saturation, effective gas permeability and NMR relaxation time distribution in two-phase fluid flow experiments. We introduce both the experimental setup and the experimental procedure on a tight Rotliegend sandstone sample. The initially fully water saturated sample is systematically drained by a stepwise increase of gas (Nitrogen) inlet pressure and the drainage process is continuously monitored by low field NMR relaxation measurements. After correction of the data for temperature fluctuations, the monitored changes in water saturation proved very accurate. The experimental procedure provides quantitative information about the total water saturation as well as about its distribution within the pore space at defined differential pressure conditions. Furthermore, the relationship between water saturation and relative (or effective) apparent permeability is directly determined.
\end{abstract}

\section{Introduction}

Petrophysical parameters play an important role in many geological applications and are subject of various research projects. For reservoir simulations, laboratory fluid flow experiments are crucial in order to determine parameters, which can be used to calculate fluid redistribution in the subsurface. Regularly, core analysis is done under ambient conditions on dry plugs or completely water saturated samples, i.e., single-phase fluid flow is measured in order to derive intrinsic permeability $[1,2]$. However, when it comes to the characterization of low permeable material (tight sandstones, shales) below the $\mathrm{mD}$-range, the experimental procedures need to be adapted for low flow rates and high fluid pressures. This is especially difficult for the determination of the effective permeability of a fluid in presence of another fluid, which can be up to three orders of magnitude lower than the intrinsic permeability [3]. In a two-phase fluid flow system, different factors have to be accounted for, which are often strongly coupled and interdependent like e.g. stress dependence, water saturation and capillary pressure. The latter is highly important, as gas flow through low permeable (partially) water saturated rocks is usually controlled by capillary pressure, i.e., as water is drained from the pores with increasing differential gas pressure [4].

In order to derive correlations between effective permeability, water saturation and capillary pressure, commonly, several experiments are carried out in different setups (core-flooding experiments, centrifuge, porous plate, mercury injection $[5,6,7,8,9]$. Generally, effective permeability experiments are conducted in different steps, each consisting of the pre-saturation and an effective permeability measurement. For each saturation level, the flow setup has to be dis- and reassembled $[10,11]$. This procedure is very time consuming and bears the risk of sample damage caused by repetitive loading / unloading cycles. Additionally, changes in water saturation during gas flow experiments cannot be directly measured in such a conventional experimental flow cell. In this work, we present a newly developed NMR flow cell, which can be loaded up to a confining pressure of $30 \mathrm{MPa}$. The apparatus allows the determination of water saturation during ongoing fluid flow experiments. Here, we introduce the experimental procedure as well as its results on an initially fully water saturated sandstone sample. After sample installation, the drainage process was continuously monitored in terms of changing water saturation and effective gas permeability. For more experimental results, obtained with the introduced NMR flow cell, we refer to [12].

\section{Theoretical Background}

\subsection{Single and Two-Phase Fluid Flow}

In a single-phase system, permeability depends only on the rock's intrinsic properties, i.e. pore size distribution and tortuosity [9]. In laboratory experiments, permeability can be determined on $\mathrm{cm}$-sized cylindrical plugs with either water or gas. When using water as the permeating liquid, Darcy's law for incompressible media is used [13]:

$$
Q=-\frac{k_{\mathrm{water}} A}{\eta} \frac{\Delta p}{\Delta x}
$$

\footnotetext{
*Corresponding author: NKlitzsch@eonerc.rwth-aachen.de
} 
Parameters are the volume flow rate $Q\left[\mathrm{~m}^{3} / \mathrm{s}\right]$, the differential pressure $\Delta \mathrm{p}[\mathrm{Pa}]$ across the sample, fluid viscosity $\eta[\mathrm{Pa} s]$, cross-sectional area $A\left[\mathrm{~m}^{2}\right]$, sample length $\Delta x[\mathrm{~m}]$ and intrinsic permeability $k_{\text {water }}\left[\mathrm{m}^{2}\right]$. When using gas in the experiments, one has to account for gas compressibility (eq. 2) and slip flow (eq. 3). Assuming the validity of the ideal gas law, integration across the sample length and between the up- and downstream pressures, $p_{1}$ and $p_{2}$, yields Darcy's law for compressible media [9]:

$$
Q=-\frac{k_{\mathrm{gas}} A}{\eta} \frac{\left(p_{1}^{2}-p_{2}^{2}\right)}{2 p_{2} \Delta x} .
$$

The coefficient $k_{\text {gas }}\left[\mathrm{m}^{2}\right]$ is the apparent gas permeability, which is only valid for given fluid pressure conditions. It is well known, that gas permeability increases with decreasing mean fluid pressures $\left(p_{\text {mean }}\right)$, which is due to the increasing molecule/pore wall interactions at low gas densities $[14,15,16]$. In singlephase flow systems, the so called "Klinkenberg" or "slip flow" correction is routinely done according to the following linear relationship with $k_{\infty}$ being the intercept on the $y$-axis, thus at infinite high gas pressure:

$$
\begin{gathered}
k_{\text {gas }}=k_{\infty}\left(1+\frac{b}{p_{\text {mean }}}\right) \\
\text { with } b=\frac{8 c p_{\text {mean }}}{d} .
\end{gathered}
$$

In eq. 4 , the slip factor $b[\mathrm{~Pa}]$ is directly related to $d[\mathrm{~m}]$, the average or mean pore diameter of the porous medium and to parameters describing the property of the gas phase $(\lambda[\mathrm{m}]$, the mean free path length of the gas molecule, $c \approx$ 1 , the Adzumi constant and $\left.p_{\text {mean }}[\mathrm{Pa}]\right)$. Accordingly, the smaller the pores, the larger the slip flow effect. In twophase systems, this is extremely difficult to measure and so far only studied for pre-defined water saturations [10, $11,17,4]$. This correction was not done here as it out of the scope of the present work.

In a two-phase fluid system, one distinguishes between the wetting and the non-wetting fluid. Both phases can be displaced by the other phase, which changes the saturation profile within the porous medium. Hereby, the terms drainage and imbibition are used, which refer to the displacement of the wetting phase by the non-wetting phase and vice versa. For most siliciclastic rocks, water is considered the wetting phase and gas the non-wetting phase. The mobility of both phases (effective permeability) is strongly coupled with fluid saturation and capillary pressure. Increasing capillary pressure results in decreasing water saturation (drainage process), which in turn results in more available fluid pathways for gas flow, thus an increased effective gas permeability. The smaller the pores, the larger is the capillary pressure, which has to be applied to drain water from the pore space. This relationship is described by the Washburn equation for the simplified model of cylindrical pores [18]:

$$
P_{\mathrm{c}}=\frac{2 \gamma \cos \theta}{R},
$$

with $P_{c}$ the capillary pressure, $\gamma[\mathrm{N} / \mathrm{m}]$ the interfacial tension between the wetting and non-wetting phase (here water and gas), $\theta\left[^{\circ}\right]$ the surface contact angle of the wetting phase and $R$ the equivalent capillary pore radius. In non-oil contaminated reservoir rocks, $\theta$ is usually assumed to be zero. In the present study, the pressures detected on the up- and downstream pressure side during the drainage experiment $\left(p_{1}, p_{2}\right)$ correspond to the pressures of the gas and water phase, respectively. Hence, in the two-phase fluid flow experiments conducted in this study, the assumption is made that $P_{\mathrm{c}}$ equals the differential pressure, $\Delta p=p_{1}-p_{2}$.

\subsection{NMR $\mathrm{T}_{2}$ relaxation measurements}

In this work, the principles of nuclear magnetic resonance (NMR) relaxation measurements are utilized to support petrophysical flow experiments - especially monitoring the change of water saturation $S_{\mathrm{w}}$, but also to qualitatively resolve the size of the corresponding water filled pores.

The NMR relaxation mechanism results from the interaction of a porous medium with its pore-filling fluid containing a detectable amount of hydrogen protons ${ }^{1} \mathrm{H}$ (here water). In most laboratory and well-logging applications, the magnetic moments (spins) of hydrogen protons are aligned with a strong static magnetic field $\boldsymbol{B}_{0}$ and therefore, yield a minute net magnetization. The characteristic precession frequency of the magnetization around the static magnetic field is called Larmor frequency $\omega_{\mathrm{L}}=\gamma_{\mathrm{H}} \boldsymbol{B}_{0}$ and depends solely on the strength of $\boldsymbol{B}_{0}$ and the gyromagnetic ratio $\gamma_{\mathrm{H}}$ of hydrogen. Larmor frequencies of commonly available applications can vary from approx. $0.2 \mathrm{MHz}(4.7 \mathrm{mT})$ to $0.4 \mathrm{GHz}(9.4 \mathrm{~T})$ [19, 20]. A NMR relaxation measurement is started by applying an energizing electromagnetic pulse with the appropriate Larmor frequency (creating a secondary electromagnetic field $\mathbf{B}_{1}$ ) and thus tipping away all spins from their equilibrium state. After the pulse is switched off, the spins relax back into their equilibrium state. This relaxation process is measured and the resulting NMR signal is given by

$$
E(t)=\sum_{i} \frac{V_{i}}{V_{0}} \exp \left(\frac{-t}{T_{2, i}}\right)
$$

with $V_{0}$ the total water filled pore volume, and $V_{i}$ the volume of pore class $i$ relaxing with the characteristic relaxation time $T_{2, i}$. The sum of individual amplitudes $\sum V_{i} / V_{0} \rightarrow E(t=0) \rightarrow E_{0}$ is commonly referred to as initial amplitude $E_{0}$ and is directly proportional to the amount of excited hydrogen protons and therewith, a direct measure for water content, and hence, saturation $S_{\mathrm{w}(\mathrm{NMR})}$.

The NMR $T_{2}$ relaxation process itself is a superposition of three independent mechanisms [19, 21]: (i) the bulk relaxation $T_{\mathrm{b}}$ of the pore fluid, (ii) the surface relaxation $T_{\mathrm{s}}$ due to the interaction of pore fluid and rock 
matrix and (iii) diffusional relaxation $T_{\mathrm{d}}$ caused by spins diffusing through a non-uniform magnetic field. In this work, we assume no internal field gradients and therefore neglect diffusional relaxation. However, in the presence of minerals that exhibit high magnetic susceptibilities, diffusional relaxation must be accounted for [22, 23, 24]. Furthermore, we also assume the so-called fast diffusion regime where $\rho_{\mathrm{s}} R / D \ll 1$, with surface relaxivity $\rho_{\mathrm{s}}$, characteristic pore size $R$ and self-diffusion coefficient of water $D$ [25]. This assumption is reasonable considering the pore sizes of the tight sandstone samples used in this study. Therefore, in a water saturated porous media the effective relaxation time $T_{2}$ is given by

$$
\frac{1}{T_{2}}=\frac{1}{T_{2, \mathrm{~b}}}+\frac{1}{T_{2, \mathrm{~s}}}+\frac{1}{T_{2, \mathrm{~d}}} \rightarrow \frac{1}{T_{2, \mathrm{~b}}}+\rho_{s} \frac{S}{V} .
$$

By inspection of eq. 7 one can deduce the following straightforward relationship: the larger the pore is, the smaller is the surface-to-volume ratio $S / V$, and hence, the longer is the relaxation time $T_{2}$ and vice versa. The surface relaxivity $\rho_{\mathrm{s}}$ is a mineral parameter and relates surface inhomogeneities to accelerated relaxation and has dimensions of velocity $\mathrm{m} / \mathrm{s}$. Generally, $\rho_{\mathrm{s}}$ is assumed constant for a particular type of porous media and needs to be determined via calibration [26]. Depending on the knowledge of $\rho_{\mathrm{s}}$ and considering eqs. 6 and 7 , it is possible to directly infer the pore size (or $S / V$ of the pore) from NMR relaxometry data.

To find the individual amplitudes $V_{i} / V_{0}$ as a function of relaxation time $T_{2, i}$ (cf. eq. 6), the so-called relaxation time distribution (RTD), a linear system of equations has to be solved. Generally, and because this inverse problem is ill-posed [27], this is achieved by a regularized (smoothed) least-square minimization [28] of the form

$$
\min \|\mathbf{G m}-\mathbf{d}\|_{2}^{2}+\lambda^{2}\|\mathbf{L m}\|_{2}^{2}
$$

with $\mathbf{d}=E(t)$ the NMR data vector, $\mathbf{m}=\left[V_{i} / V_{0}\right]$ the model vector and the forward operator $\mathbf{G}$ (cf. eq. 6). Here, the smoothness constraint on $\mathbf{m}$ is applied by a first-order derivative matrix $\mathbf{L}$. The regularization parameter $\lambda$ is found via the L-curve criterion and chosen such that the inversion misfit is in the order of the data noise while keeping a sufficiently smooth RTD [29, 28].

\section{Experimental Methods \& Procedures}

\subsection{NMR Flow Cell Setup}

The experimental setup used in this study consists of multiple components, which allow for the combined measurement of fluid flow and NMR. An overview of the complete assembly is given in Fig. 1. The high-pressure flow cell, which is designed for samples of $30 \mathrm{~mm}$ in diameter and $15-60 \mathrm{~mm}$ in length, is placed vertically in the center of a Halbach magnet. The sample itself is placed between two NMR-inert PEEK (polyether ether ketone) pistons containing conduits for in- and outflow. Grooves on the pistons allow for the even distribution of the gas phase across the sample surface. A rubber sleeve encases the piston/sample arrangement. O-rings prevent influx of the confining pressure oil. The RF coil, which is a $13 \mathrm{~mm}$ long copper radio frequency coil (rf-coil), is located within the confining compartment surrounding the rubber sleeve and is centrally positioned around the sample plug. In order to apply a constant confining pressure, $p_{\text {conf }}$, (up to $30 \mathrm{MPa}$ ) the compartment is filled with a NMR-inert synthetic oil (Fluorinert ${ }^{\mathrm{TM}}$ FC-40), regulated by a HPLC pump (Shimadzu LC-6A). Temperature fluctuations within the flow cell are monitored with a resistance thermometer PT 100 with an accuracy of $0.05 \mathrm{~K}$. On the outside, a wooden cylinder encases the entire setup.

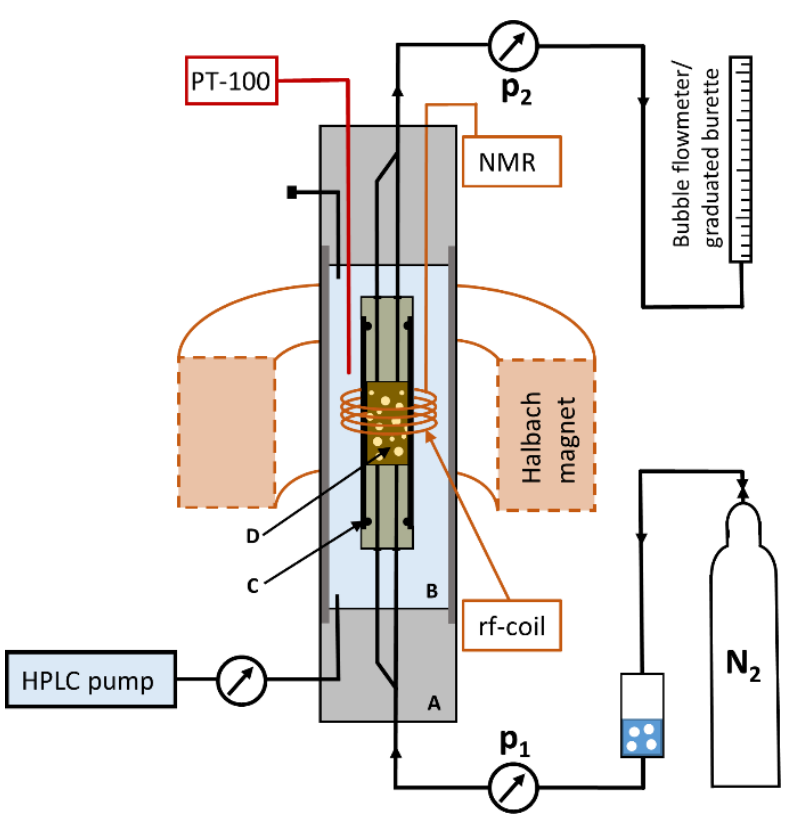

Figure 1. Sketch of the NMR flow cell with A - outer sealing system, B - confining pressure compartment filled with NMRinert oil, C - inner O-ring sealed sleeve system, D - sample placed between PEEK pistons.

The flow cell is connected to a nitrogen gas bottle on the high-pressure side $\mathrm{p}_{1}$ at the base of the setup. The lowpressure side $\mathrm{p}_{2}$ is kept at atmospheric pressure, i.e., the gas flows opposite to gravity. In this system, gravitational forces on the water phase can be neglected as they are much smaller than the applied differential gas pressures of at least $100 \mathrm{kPa}$. A water reservoir moisturizes the gas stream in order to prevent drying of the sample. The downstream capillary is connected to either a bubble flow meter for gas flux measurements or a graduated pipette to determine single-phase water flux (top of the setup). Additionally, two pressure transducers (Keller, 0.05\% FSO) at the inlet/outlet of the flow cell continuously monitor the pressure on both sides.

\subsection{NMR Data Processing}

All NMR relaxation measurements presented in this work are conducted with a low field Halbach NMR setup working at a frequency of $4 \mathrm{MHz}[30,31]$ and using the 
Carr-Purcell-Meiboom-Gill (CPMG) pulse sequence [32, 33]. The shortest possible echo time in our setup is $T E=$ $320 \mu s$ and, depending on the saturation and relaxation behavior of the corresponding sample, up to 2500 echoes were recorded, yielding signal lengths up to $0.8 \mathrm{~s}$. Initially, each CPMG echo train was averaged until a signal-to-noise ratio of $S / N \sim 200$ was reached. Over the course of the drainage experiment and due to the decreasing water saturation (and hence, decreasing signal amplitude), this constraint could not be maintained while keeping an equal NMR measurement interval of 30 minutes.

According to Curie's law the magnetization is negatively correlated with temperature, i.e., for water, a temperature decrease of $1 \mathrm{~K}$ yields a magnetization increase of $\sim 0.4 \%$ and vice versa. However, in preliminary experiments we found, that even small temperature fluctuations have a much stronger effect on the initial amplitude of the NMR signal as expected. For testing purposes, we installed a $100 \%$ water filled dummy sample (PVC container filled with degassed tap water) in the flow cell and the confining compartment was filled with the NMR-inert oil - the confining pressure was allowed to be atmospheric. Like in a drainage experiment, the temperatures inside the flow cell $T_{\text {cell }}$ and the laboratory $T_{\text {room }}$ as well as the initial NMR amplitudes $E_{0}$ were continuously monitored over a period of 30 hours. From Fig. 2 a strong positive correlation between the NMR amplitude $\left(\mathrm{NMR}_{\text {raw }}\right)$ and the temperature measured within the flow cell $\left(T_{\text {cell }}\right)$ is evident $\left(R^{2}=0.9990\right.$, cf. inset in Fig. 2). While the temperature inside the flow cell $T_{\text {cell }}$ (solid black line) varied by approximately $\pm 0.1 \mathrm{~K}$, the initial amplitude varied by about \pm 10 [a. u. ] or $4 \%$. This value is two orders of magnitude higher than the theoretical increase. As the NMR amplitude correlates linearly with the temperature fluctuation (see inset of Fig. 2), the latter can be used to correct the NMR amplitudes. The temperature-corrected signal $\left(\mathrm{NMR}_{\text {corr }}\right)$ fluctuates less than $0.2 \%$ around its mean value. For this reason, we conduct this pre-test always on the fully water saturated samples before starting the actual drainage experiment. We found, that a measurement time of approx. 48 hours yielded stable correlations between NMR amplitude and temperature fluctuations.

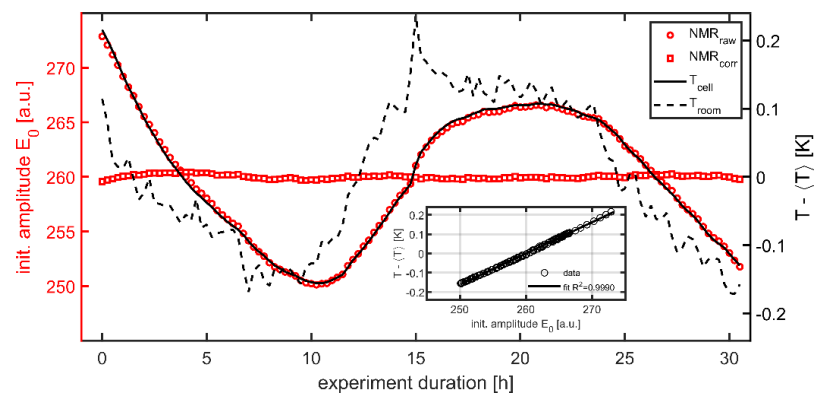

Figure 2. Raw (red circles) and corrected (red squares) initial NMR amplitudes E0 together with the flow cell (black solid) and room temperature (black dashed) variations for a water sample; $\langle\mathrm{T}\rangle$ denotes the mean temperature of the flow cell or the room, respectively. The inset shows the correlation between the raw amplitude and cell temperature fluctuation.
The effect of temperature on bulk relaxation is well understood and can easily be approximated [19]. In the case of our test measurement with $100 \%$ water, the measured bulk relaxation time follows the theoretical relation. Unlike modern NMR devices, our setup is not temperature regulated. We hypothesize that the temperature dependence of the NMR amplitude observed in our measurements is device or setup dependent, e.g., temperature effects on the electronic components of the resonant circuit. With the procedure described above, we are able to account for the temperature fluctuations and derive reliable water saturation information from our experiment.

\subsection{Single-Phase Flow - Intrinsic Gas Permeability}

To qualitatively and quantitatively compare single-phase and two-phase flow results, we use for both types of measurements the NMR flow cell under equal confining pressure conditions. To determine the intrinsic permeability $k_{\infty}$, the dry sample is installed into the flow cell and the capillaries on the high-pressure side are connected to a nitrogen gas source. After application of the confining pressure, the gas permeability experiments are conducted at different mean fluid pressures $p_{\text {mean }}$. Here, steady state experiments are conducted at different upstream pressures $p_{1}$, while keeping the downstream pressure $p_{2}$ at ambient conditions. The volume flow $Q$, is measured on the outflow side with the attached bubble flow meter until steady state flow conditions are established. Apparent and intrinsic permeability values are determined from eqs. 2 and 3.

\subsection{Two-Phase Flow - Effective Gas Permeability}

Prior to all drainage experiments, air is removed from the sample in a vacuum desiccator. Thereafter, we saturated the sample with brine (10.6 g/L $\mathrm{MgSO}_{4}$ solution) and installed it into the flow cell. There, a confining pressure of $15 \mathrm{MPa}$ is established. To obtain a sufficient amount of data for the temperature correction described above, repetitive NMR measurements (30 min interval) are performed on the fully water saturated and pressurized sample for a time span of at least 48 hours.

Drainage experiments are performed according to the step-wise procedure usually applied for gas breakthrough determination of sealing lithologies [34]. The drainage experiment is conducted by increasing the gas pressure on the high-pressure side in different intervals $\left(p_{1}=0.3-\right.$ $2.5 \mathrm{MPa})$, while the outflow side is constantly held at atmospheric pressure $\left(p_{2}=\right.$ const.). At low differential pressures, only water is displaced from the sample but no gas flows through it. After exceeding a threshold pressure, which equals the capillary pressure of the smallest pore along the percolation path, the gas phase breaks through and a saturation gradient establishes along the sample, i.e., along the pressure gradient. The whole drainage experiment is monitored by NMR measurements (30 min interval), as well as temperature and pressure measurements (30 s interval). Because of the saturation gradient and the sensitive range of the NMR coil of about 
$2 \mathrm{~cm}$, we measure an average water saturation over this range. First gas flow being detected on the low-pressure side indicates that the capillary breakthrough pressure has been overcome. At this point, sufficient water has been displaced from the pore space and at least one gas conducting percolation path has been established. The number of percolation pathways increases with increasing gas pressure. Moreover, more pores are desaturated at the high-pressure side than at the low-pressure side, i.e., there is a saturation gradient, which changes with increasing gas pressure. Regularly, the gas outflow rate is detected with a bubble flow meter. After gas outflow and NMR signal (saturation) stabilize, the next pressure difference is applied. From the bubble flow meter and from the NMR data at stationary conditions, we calculate effective permeability of the gas phase, water saturation and relaxation time distribution, respectively. They in turn can be related to the applied pressure difference.

\section{Results \& Discussion}

\subsection{Sample Characterization \& Intrinsic Properties}

The test plug (R1), used in this study, was provided by the Wintershall Holding $\mathrm{GmbH}$ and is a tight reservoir sandstone from a depth of about $4000 \mathrm{~m}$. It is a coarsegrained sandstone from the Rotliegend formation, containing illite as pore filling mineral. Some petrophysical properties of sample R1 are provided in Tab. 1 .

Table 1. Petrophysical properties (logarithmic mean NMR relaxation time $T_{\mathrm{lgm}}$, NMR porosity $\Phi_{\mathrm{NMR}}$, Helium porosity $\Phi_{\mathrm{He}}$, Archimedes porosity $\Phi_{\text {Arch }}$, intrinsic gas permeability $\mathrm{k}_{\infty}$, and Klinkenberg slip factor $b$ ) of the sample used in this study.

\begin{tabular}{|c|c|c|c|}
\hline $\mathrm{T}_{\operatorname{lgm}}[\mathrm{ms}]$ & \begin{tabular}{|}
$\boldsymbol{\Phi}_{\mathrm{NMR}}\left|\boldsymbol{\Phi}_{\mathrm{He}}\right| \boldsymbol{\Phi}_{\mathrm{Arch}}[\%]$ \\
\end{tabular} & $\mathbf{k}_{\infty}\left[\mathbf{m}^{2}\right]$ & $b[\mathrm{MPa}]$ \\
\hline 4.8 & \begin{tabular}{l|l|l|l}
5.8 & 6.7 & 6.9 \\
\end{tabular} & $4 \times 10^{-17}$ & 0.20 \\
\hline
\end{tabular}

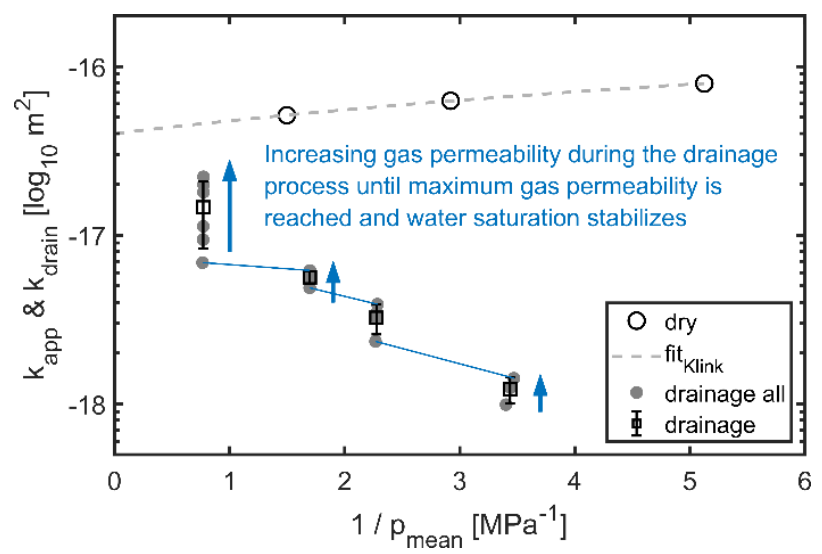

Figure 3. Apparent gas permeability for the dry R1 sample (circles) and effective gas permeability during drainage (squares) as a function of reciprocal mean pressure pmean. The dashed line is a linear fit to the dry data yielding an intrinsic gas permeability of $k_{\infty}=410^{-17} \mathrm{~m}^{2}$.

Porosity was determined via Archimedes' principle. The intrinsic (Klinkenberg corrected) permeability is $410^{-17} \mathrm{~m}^{2}$ and the slip factor is $0.2 \mathrm{MPa}$ (cf. Tab. 1). In
Fig. 3, the apparent permeability $k_{\text {app }}$ as a function of reciprocal mean pressure $p_{\text {mean }}$ is shown (black circles). The Klinkenberg fit to derive the intrinsic permeability $k_{\infty}$ is depicted with the gray dashed line.

In Fig. 4 a typical NMR measurement is shown. The NMR transient (solid gray line) and the corresponding multi-exponential fit (solid black line) are depicted in Fig. 4a. The signal consists of 750 echoes with an inter-echo time of $320 \mu \mathrm{s}$. The signal-to-noise ratio (SNR) of this particular measurement is 180 . Figure $4 \mathrm{~b}$ shows the corresponding inverted relaxation time distribution (RTD). The RTD was derived as described above using a smoothed least squares fit with 20 relaxation times per decade. The regularization parameter was chosen as such, that the fitting error $(\mathrm{rms}=0.07)$ is equal to the noise level of the data (dashed black line in Fig. 4a). The corresponding solid gray line in Fig. $4 \mathrm{~b}$ represents the cumulative relaxation time distribution. The vertical black dashed line in Fig. 4b indicates the characteristic relaxation time of the RTD often referred to as logarithmic mean relaxation time $\mathrm{T}_{\text {lgm }}$.
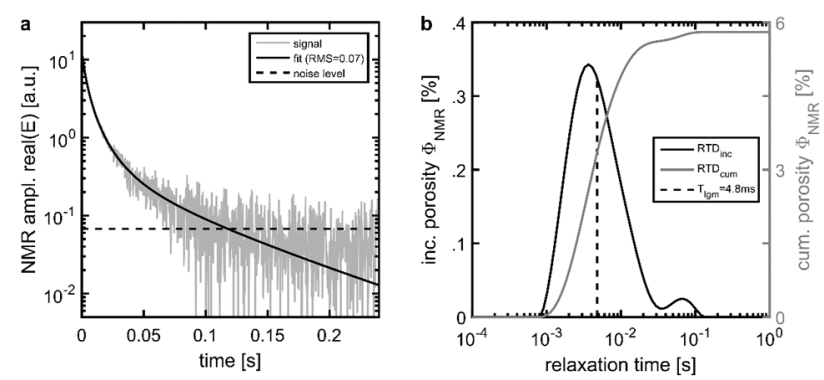

Figure 4. (a) T2 NMR signal (gray solid) and corresponding fit (black solid) for the full saturated sample R1; (b) corresponding incremental (black solid) and cumulative (gray solid) relaxation time distribution.

The RTD for sample R1 is characterized by one pronounced peak at about $4 \mathrm{~ms}$ and a minor peak at about 70 ms. Following the standard classification of RTDs [19] more than $95 \%$ of the signal originates from pores with relaxation times smaller than $33 \mathrm{~ms}$ and hence, classifies as bulk volume irreducible (BVI) and clay bound water $(\mathrm{CBW})$. This already indicates the tight character of the sample.

A pre-characterization of the intrinsic properties before conducting the actual drainage experiment is generally advisable. Thereafter, the experimental protocol can be adjusted accordingly, i.e., with respect to the choice of initial pressure difference or the amount of pressure steps. If, for instance, samples exhibit a narrow pore size distribution, only a few (densely spaced) pressure steps can be applied before the irreducible water saturation is reached.

\subsection{Drainage Experiment}

The drainage experiment for sample $\mathrm{R} 1$ was conducted at $15 \mathrm{MPa}$ confining pressure. The differential pressure ranged from 0.2 to $2.4 \mathrm{MPa}$ and the sample was drained to a $S_{w, i r r}$ of 0.13 . The temperature over the course of the 
entire experiment was $\left(19.97 \pm 0.21^{\circ} \mathrm{C}\right)$.

During the drainage experiment, we applied five distinct pressure steps (cf. Fig. 5a). At the first differential pressure of $0.2 \mathrm{MPa}$, no significant saturation decrease and no gas flow could be measured over the course of $24 \mathrm{~h}$. Effective gas permeabilities were measured for the first time once breakthrough occurred and gas flow was established. This happened after approx. $50 \mathrm{~h}$ at a differential pressure of $0.4 \mathrm{MPa}$. In the following, differential pressure was increased approx. every $24 \mathrm{~h}$ depending on the equilibration of water saturation and effective gas permeability.

The temperature fluctuation inside the flow cell is depicted in Fig. 5b. The fluctuations show amplitudes of $\pm 0.5 \mathrm{~K}$ over the course of the experiment and decrease towards the end of the experiment. Fig. 5c shows the raw and temperature-corrected NMR amplitudes in gray and black, respectively. There are several events (Fig. 5b, c) where the change of temperature clearly influences the NMR amplitudes. For instance, between $24 \mathrm{~h}$ and $72 \mathrm{~h}$ the temperature varied between $-0.5 \mathrm{~K}$ and $0.5 \mathrm{~K}$ yielding a saturation fluctuation of $\pm 10 \%$. Figure $5 \mathrm{c}$ visualizes the impact of the aforementioned temperature correction. Without correction, water saturation (raw in Fig. 5) increases after the onset of gas flow ( $\geq 48 \mathrm{~h}$ ), which is clearly unphysical considering the given experimental setup, but slightly decreases after temperature correction (corr. in Fig. 5). Effective gas permeabilities increased by more than an order of magnitude during the entire measuring cycle from $9.910^{-19} \mathrm{~m}^{2}$ to $2.210^{-17} \mathrm{~m}^{2}$ at differential pressures of $0.4 \mathrm{MPa}$ and $2.4 \mathrm{MPa}$, respectively.

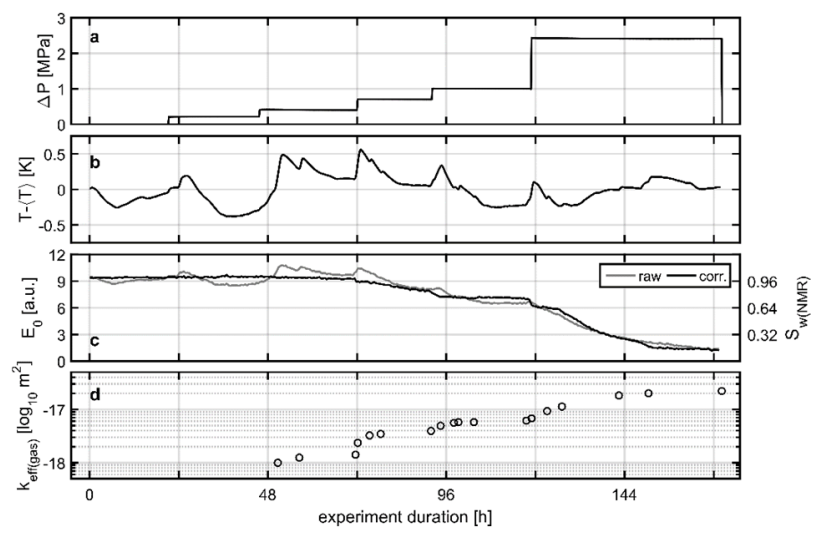

Figure 5. Parameters recorded during the drainage experiment: (a) differential pressure; (b) temperature fluctuation inside flow cell; (c) raw (gray) and temperature-corrected (black) initial NMR amplitude or water saturation; (d) effective gas permeability.

The increase of effective gas permeability with increasing differential pressure clearly occurs due to the drainage of successively smaller pores. This process can be visualized by contiguously plotting the RTD in a carpet plot, see panel $\mathbf{I}$ in Fig. 6. Even though we think that the drainage of successively smaller pores dominates the observed RTD changes, the shift towards shorter relaxation times with decreasing saturation might also be caused by the associated changes in the surface-to-volume ratio of drained pores. For example, water trapped in the corners of a desaturated angular pore contributes to the RTD with considerably smaller relaxation times than the originally saturated pore [35]. Panel $\mathbf{I}$ in Fig. 6 shows the relative amplitudes of the RTD (incremental porosity cf. Fig. 4b) ranging from $0.005 \%$ (white) to $0.35 \%$ (black) over the entire duration of the experiment. For better visualization, all amplitudes smaller than $0.005 \%$ are not shown. The orange points in panel $\mathbf{I}$ indicate the logarithmic mean relaxation time $T_{\operatorname{lgm}}$ for every individual relaxation time distribution. It shows the change of the (logarithmic) mean size of the water saturated pores over the course of the experiment. For reference, the corresponding full saturation relaxation time distribution is shown in panel II. In panel III the temperature-corrected saturation $S_{\mathrm{w}(\mathrm{NMR})}$ is plotted, which is given by the ratio of the initial NMR amplitudes $E_{0}(t>0)$ to the first initial NMR amplitude at full saturation $E_{0}(t=0)$.

During the first $48 \mathrm{~h}$ of the drainage experiment, the saturation stays constant and all relaxation time distributions are very similar (cf. Fig. 4b). Like for the saturation, there is no visible change of relaxation times at the first pressure step of $0.2 \mathrm{MPa}$ (after $22 \mathrm{~h}$ ), i.e., the pressure is not high enough to establish a percolation pathway in the pore network. When increasing the differential pressure to $0.4 \mathrm{MPa}$ (between $48-72 \mathrm{~h}$ ) gas breakthrough is observed and saturation, as well as $T_{\text {lgm }}$, decrease only slightly. At $0.7 \mathrm{MPa}$ differential pressure $(72-90 \mathrm{~h})$, saturation and $T_{\text {lgm }}$ decrease significantly mainly due to the drainage of larger pores $(>20 \mathrm{~ms})$. After a first strong decrease in saturation and $T_{\operatorname{lgm}}$ at $1 \mathrm{MPa}$ differential pressure the decrease levels off and all values stay constant for approximately $24 \mathrm{~h}$. The final increase to $2.4 \mathrm{MPa}$ differential pressure yields a rather strong drainage effect and a final water saturation of $S_{\mathrm{w}(\mathrm{NMR})}=0.13$. Here, the strong drainage relates to pores having relaxation times between $1-10 \mathrm{~ms}$. We cross-checked the final saturation by weighing the sample after the experiment and it was found to be $S_{\mathrm{w} \text { (weigh) }}=$ 0.15 , so comparable to the value derived from the NMR measurement. 


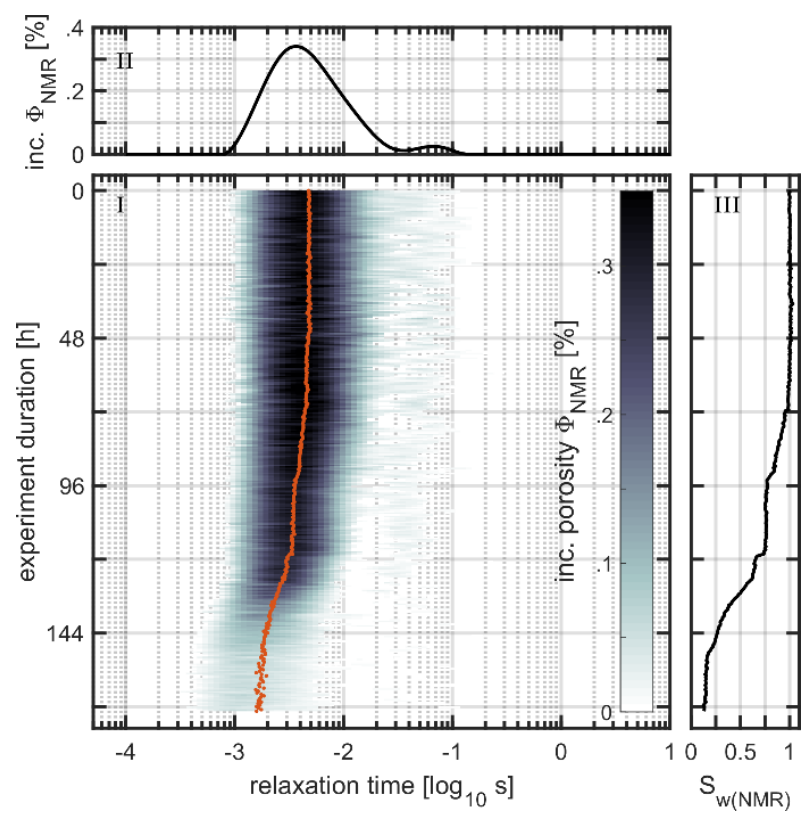

Figure 6. panel (I) RTDs over experiment duration; panel (II) full saturation RTD; panel (III) water saturation over experiment duration; orange points in panel (I) refer to the logarithmic mean relaxation time $T_{\mathrm{lgm}}$.

\subsection{History Matching}

The simultaneous recording of average water saturation (water production) and pressure gradient throughout a drainage experiment allows to history match recorded data by a two-phase flow simulator. A $1 \mathrm{D}$ model is used for the simulation assuming a homogeneous rock in all three dimensions. Software packages available are similar to reservoir black-oil simulators [36] and based on immiscible displacement of the fluid phases [37, 38]. They fit experimental data at distinct time steps, e.g., pressure gradient and water production, by adjusting capillary pressure and relative permeability as these curves are related to pressure and saturation. Analytical functions as Corey (power law), LET, Log (beta) etc. or measured data can be selected. In this study the software $\mathrm{Cydar}^{\odot}$ [39] was used to history match the data. In order to constrain the number of solutions, the measured relative gas permeability curve was fitted using Corey exponents and considered invariable throughout the history match. The history match was run by simulating the NMR water saturations and pressure recordings and adjusting the analytical curves of the relative permeability of water and the drainage capillary pressure. Figure 7 shows a comparison of the measured and simulated water production, the measured and simulated pressure gradients and the relative permeability curves of gas and water, as well as the drainage capillary pressure data. The capillary pressure function includes an entry pressure $\left(P_{e}\right)$ where gas starts to enter the pore space and displaces the water phase. The value of this entry pressure is consistent with the experimental differential pressure where a decrease of the NMR water saturation was observed. The analytical functions simulating the experimental data in $1 \mathrm{D}$ are according to $[40,41]$.

$$
\begin{gathered}
S_{w n}=\left(S_{w}-S_{w i r r}\right) /\left(1-S_{w i r r}\right), \\
P_{c}=P_{e}-\alpha \log S_{w n}, \\
k_{r w}=k_{r w 0}\left(S_{w n}\right)^{N w}, \\
k_{r g}=k_{r g 0}\left(1-S_{w n}\right)^{N g},
\end{gathered}
$$

with $S_{w}$ the measured water saturation, $S_{\text {wirr }}$ the residual water saturation, $P_{e}$ the pore entry pressure, $\alpha$ as fitting parameter, $k_{r w}$ and $k_{r g}$ the relative permeabilities of water and gas respectively, and $\mathrm{Nw}$ and $\mathrm{Ng}$ the Corey fitting parameters.

Figure $7 \mathrm{a}+\mathrm{b}$ show the history match results for water saturation and differential pressure from the drainage experiment of sample R1. In Fig. 7c, the measured relative permeability (circles) is plotted. The fitted relative permeability for gas (dashed lines) and water (solid lines) are derived from history matching the monitored water saturation, pressure gradient and effective permeabilities. For the presented data, the derived Corey parameters yield a robust estimation of relative gas permeability over the entire water saturation range. Additionally, capillary pressure data can also be obtained from the history match, as shown in Fig. 7d.
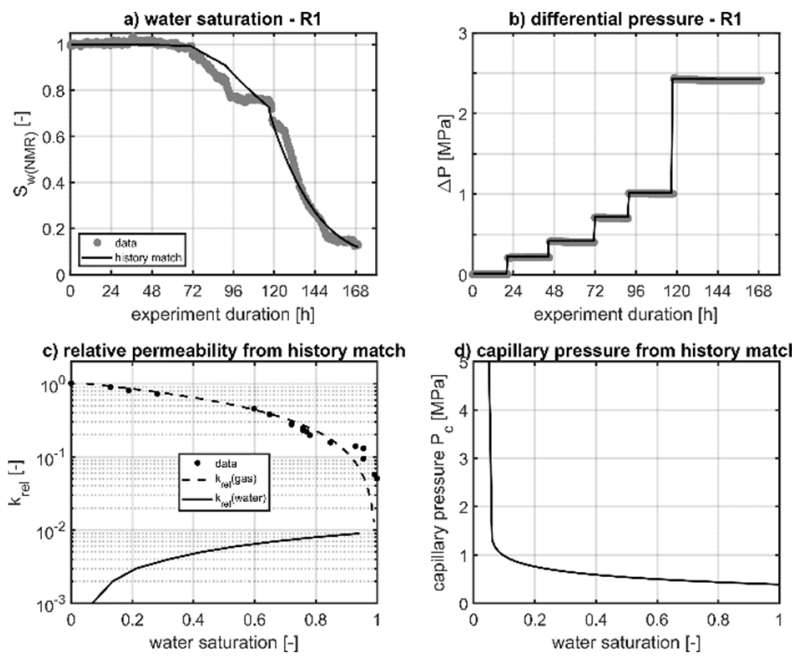

Figure 7. (a) History matched water saturation and (b) differential pressure for sample R1; (c) estimated relative permeabilities and (d) capillary pressure derived from history matching the monitored data for all four samples. The circles in panel $\mathrm{a}, \mathrm{b}$ and $\mathrm{c}$ show the corresponding experimental data.

\section{Conclusion}

With the combined NMR and fluid flow measurements, we overcome the lengthy experimental series of different experiments that are generally used to characterize fluid flow properties of rocks (repetitive pre-saturation tests and permeability experiments). The test measurements proved crucial and resulted in significant improvements for the workflow and thus data quality.

The combination of NMR relaxation measurements and two-phase fluid flow experiments allows for the continuous allocation of water saturation $S_{\mathrm{w}(\mathrm{NMR})}$ to differential pressure $\Delta p$ (respectively capillary pressure 
$P_{\mathrm{c}}$ ). Moreover, it provides information about the changing water distribution within the pore space, i.e., about the size of the water filled pores, over the course of the experiment. Furthermore, with the our NMR flow cell setup, we directly measure the relationship between water saturation and effective gas permeability $k_{\text {eff(gas) }}$.

After accounting for temperature fluctuations, the monitored changes in water saturation proved very accurate. However, for future experiments, it may be advantageous to place the whole setup in a temperaturecontrolled container.

The data recorded with the NMR flow cell, can be utilized for the parametrization of the relations between effective permeability, water saturation and capillary pressure by an inverse simulation of the experiment, i.e., by a history match. Hence, the results can readily enter a reservoir simulation.

The authors gratefully acknowledge Wintershall Holding GmbH for funding the iLoPS research project, within this study was conducted. We also like to thank Bernhard Krooss, Jonas Kaiser and Lena Kämpfner for their valuable input and help with the experimental setup.

\section{References}

1 E.J. Peters, Advanced Petrophysics: Geology, Porosity, Absolute Permeability, Heterogeneity and Geostatistics, 1, Live Oak Book Company (2012)

2 M. Miller, B. Lieber, G. Piekenbrock and T. McGinness, "Low Permeability Gas Reservoirs How Low Can You Go?" SPWLA Middle East Regional Symposium (2007)

3 K.W. Shanley, R.M. Cluff and J.W. Robinson, "Factors controlling prolific gas production from lowpermeability sandstone reservoirs: Implications for resource assessment, prospect development, and risk analysis," AAPG bulletin, 88, 1083-1121 (2004)

4 A. Amann-Hildenbrand, J.P. Dietrichs and B.M. Krooss, "Effective gas permeability of Tight Gas Sandstones as a function of capillary pressure-a non-steady-state approach," Geofluids, 16, 367-383 (2016)

5 W.R. Purcell, "Capillary Pressures - Their Measurement Using Mercury and the Calculation of Permeability Therefrom," Trans., AIME 186, 39-48 (1949)

6 J.S. Osoba, J.G. Richardson, J.K. Kerver, J.A. Hafford and P.M. Blair, "Laboratory Measurements of Relative Permeability," Trans., AIME 192, 47-56 (1951)

7 M.M. Honarpour, F.Koederitz and A. Herbert, Relative permeability of petroleum reservoirs, CRC Press Inc, Boca Raton, FL (1986)

8 C.L. Vavra, J.G. Kaldi and R.M. Sneider, "Geological applications of capillary pressure: a review (1)," AAPG Bulletin, 76, 840-850 (1992)

9 D. Tiab and E.C. Donaldson, Petrophysics: Theory and Practice of Measuring Reservoir Rock and Fluid Transport Properties, 4th ed., Waltham (USA): Gulf Professional Publishing (2015)

10 R.K. Estes and P.F. Fulton, "Gas slippage in permeability measurements," J. Pet. Tech, 8, 69-73 (1956)
11 J.A. Rushing, K.E. Newsham and V.a.K.C. Fraassen, "Measurement of the Two-Phase Gas Slippage Phenomenon and its Effect on Effective Gas Permeability in Tight Gas Sands," SPE Annual Technical Conference and Exhibition (2003)

12 A. Amann-Hildenbrand, M. Shabani, T. Hiller, N. Klitzsch, N. Schleifer and B. M. Krooss, " Gas Slippage in Partially Saturated Tight Rocks and During Drainage," SCA2019, \#79 (2019)

13 H.P.G. Darcy, Les Fontaines publiques de la ville de Dijon, Victor Dalamont (1856)

14 A. Kundt, E. Warburg, "Über Reibung und Wärmeleitung verdünnter Gase," Annalen der Physik, 232, 177-211 (1875)

15 M. Knudsen, "Die Gesetze der Molekularströmung und der inneren Reibungsströmung der Gase durch Röhren," Annalen der Physik, 333, 75-130 (1909)

16 L.J. Klinkenberg, "The permeability of porous media to liquids and gases," Drilling and production practice (1941)

17 L. Qingjie, L. Baohua, L. Xianbing, Y. Shouguo, "The effect of water saturation on gas slip factor by pore scale network modeling," SCA 2002 Symposium (2002)

18 E.W. Washburn, "The dynamics of capillary flow," Phys. Rev., 17(3),. 273-283 (1921)

19 G.R. Coates, L. Xiao, M.G. Prammer, NMR logging: principles and applications (1999)

20 A.A. Behroozmand, K. Keating and E. Auken, "A review of the principles and applications of the NMR technique for near-surface characterization," Surveys in Geophysics, 36, 27-85 (2015)

21 K.J. Dunn, D.J. Bergman and G.A. LaTorraca, Nuclear magnetic resonance: petrophysical and logging applications (2002)

22 K. Keating and R. Knight, "A laboratory study of the effect of magnetite on NMR relaxation rates," J. Appl. Geophys., 66, 188-196 (2008)

23 K. Keating and R. Knight, "A laboratory study of the effect of Fe (II)-bearing minerals on nuclear magnetic resonance (NMR) relaxation measurements," Geophysics, 75, F71-F82 (2010)

24 J. Mitchell, T.C. Chandrasekera, M.L. Johns, L.F Gladden and E.J. Fordham, "Nuclear magnetic resonance relaxation and diffusion in the presence of internal gradients: The effect of magnetic field strength," Phys. Rev. E, 81(2), 026101 (2010)

25 K.R. Brownstein and C.E. Tarr, "Importance of classical diffusion in NMR studies of water in biological cells," Phys. Rev. A, 19(6), 2446-2453 (1979)

26 W.E. Kenyon, "Petrophysical principles of applications of NMR logging," The Log Analyst, 38(2), 21-43 (1997)

27 J. Hadamard, Lectures on Cauchy's problem in linear partial differential equations, Yale University Press, New York (1923)

28 R.C. Aster, B. Borchers, C.H. Thurber, Parameter Estimation and Inverse Problems, 2nd ed. (2013)

29 P.C. Hansen, Rank-Deficient and Discrete Ill-Posed Problems: Numerical Aspects of Linear Inversion, SIAM Philadelphia, (1998)

30 K. Halbach, "Design of permanent multipole magnets with oriented rare earth cobalt material," Nucl. Instrum. Methods, 169, 1-10 (1980) 
31 S. Anferova, V. Anferov, J. Arnold, E. Talnishnikh, M.A. Voda, K. Kupferschläger, P. Blümler, C. Clauser and B. Blümich, "Improved Halbach sensor for NMR scanning of drill cores," Mag. Res. Imag., 25, 474-480 (2007)

32 H.Y. Carr and E.M. Purcell, Phys. Rev., 94(3), 630-638 (1954)

33 S. Meiboom and D. Gill, "Effects of diffusion on free precession in nuclear magnetic resonance experiments,' Rev Sci. Instrum., 29(8), 688-691 (1958)

34 P. Egermann, J.M. Lombard and P. Bretonnier, "A fast and accurate method to measure threshold capillary pressure of caprocks under representative conditions," International Symposium of the Society of Core Analysts (2006)

35 O. Mohnke, R. Jorand, C. Nordlund and N. Klitzsch, "Understanding NMR relaxometry of partially watersaturated rocks," Hydrol. Earth Syst. Sci., 19, 2763-2773 (2015)

36 K. Aziz and A. Settari, Petroleum reservoir simulation (1979)

37 E.F. Johnson, D.P. Bossler and V.O. Naumann, "Calculation of relative permeability from displacement experiments," Pet. Trans., AIME, 216, 370-372 (1959)

38 S.C. Jones and W.O. Roszelle, "Graphical techniques for Determining Relative Permeability from Displacement Experiments," J. Pet. Tech., 30, 807-817 (1978)

39 R. Lenormand and G. Lenormand, Cydar@ User Manual, Cydarex@ Company Brochure (2016)

40 A.T. Corey, "The interrelation between gas and oil relative permeabilities," Producers monthly, 19, 38-41 (1954)

41 R.G. Bentsen and J. Anli, "Using parameter estimation techniques to convert centrifuge data into a capillarypressure curve,” Soc. Pet. Eng. J., 17, 57-64 (1977) 